JOURNAL CLUB

\title{
Fibulin-3 as a blood and effusion biomarker for pleural mesothelioma
}

This study investigated whether plasma and pleural effusion levels of fibulin-3 (an extracellular glycoprotein found to be expressed by mesothelioma cells) could be used to differentiate pleural mesothelioma from other pleural effects of asbestos exposure; benign pleural effusions, pleural effusions from other malignancy and healthy controls. Previously identified biomarkers have shown unacceptable sensitivity and specificity.

In two cohorts plasma fibulin-3 levels were significantly higher in mesothelioma patients than in the other groups. An overall comparison of patients (with and without mesothelioma) showed that the receiver-operating-characteristic curve had a sensitivity of $96.7 \%$ and specificity of $95.5 \%$. Importantly, levels did not vary according to age, sex, duration of asbestos exposure or radiographic change.

Fibulin-3 levels in mesothelioma related pleural effusions were also shown to be significantly higher than those effusions from other causes (including other malignancies). Samples from both cohorts were identified prospectively and it is not clear why only some patients had plasma fibulin-3 levels measured. Blinded validation was performed with plasma specimens from a separate cohort.

Compared with previous attempts to identify biomarkers these results show promise. However, numbers in this study are small and there appears to have been selection bias in the samples analysed. If larger international studies can validate these findings then it has important implications for the role of early surgical or medical intervention in mesothelioma, and also for the financial compensation available to patients.

- Pass HI, Levin SM, Harbut MR, et al. Fibulin-3 as a blood and effusion biomarker for pleural mesothelioma. N Engl J Med 2012;367:1417-27.

James Murray

Correspondence to Dr James Murray, ST3, Department of Respiratory Medicine, King George Hospital, Barley Lane, Ifford, London IG3 8YB, UK; jamesmurray@doctors.org.uk

To cite Murray J. Thorax 2013;68:1180.

Published Online First 7 March 2013

Thorax 2013;68:1180. doi:10.1136/thoraxjnl-2013-203396 